\title{
Exciton dissociation and migration in enhanced order conjugated polymer/nanoparticle hybrid materials
}

\author{
Yun-Yue Lin ${ }^{1}$, Chun-Wei Chen ${ }^{1,3}$, J Chang ${ }^{2}$, T Y Lin ${ }^{2}$, I S Liu ${ }^{1}$ and \\ Wei-Fang $\mathrm{Su}^{1}$ \\ ${ }^{1}$ Department of Materials Science and Engineering, National Taiwan University, Taipei, \\ Taiwan, Republic of China \\ ${ }^{2}$ Institute of Optoelectronic Sciences, National Taiwan Ocean University, Keelung, Taiwan, \\ Republic of China \\ E-mail: chunwei@ntu.edu.tw
}

Received 3 October 2005, in final form 6 December 2005

Published 7 February 2006

Online at stacks.iop.org/Nano/17/1260

\begin{abstract}
The mechanisms of exciton dissociation and migration in the conjugated polymer (poly(2-methoxy-5-(2'-ethyl)(hexyloxy)1,4-

phenylenevinylene))(MEH-PPV)/CdSe nanoparticle hybrid materials were investigated by steady-state and time-resolved photoluminescence spectroscopy. Rapid exciton dissociation at the nanoparticle/polymer interfaces, leading to quenching of the photoluminescence efficiency $\eta$ and shortening of the measured lifetime $\tau_{\mathrm{PL}}$, is observed. The excitons which contribute to the remaining luminescence in the polymer migrate to the lower energy sites with longer conjugated sequences in the composites. The result is further evident from the observation of a redshift of the photoluminescence peak positions, a progressive decrease of the Huang-Rhys factor $S$ and an increase in the natural radiative lifetime $\tau_{R}$ with increasing $\mathrm{CdSe}$ nanoparticle content.
\end{abstract}

(Some figures in this article are in colour only in the electronic version)

\section{Introduction}

Recently, composites of organic polymers and inorganic nanoparticles have attracted great interest due to their potential application in the development of low cost, large area, mechanically flexible photovoltaic devices $[1,2]$. A basic requirement for a photovoltaic material is to generate free charge carriers produced by photoexcitation. Subsequently, these carriers are transported through the device to the electrodes without recombining with oppositely charged carriers. Due to the low dielectric constant of organic materials, the dominant photogenerated species in most conjugated polymers is a neutral bound electron-hole pair (exciton). These neutral excitons can be dissociated from Coulomb attraction by offering an energetically favourable pathway for the electron to transfer onto to an electronaccepting species. Therefore, charge separation in the polymer is often enhanced by inclusion of a high electron

3 Author to whom any correspondence should be addressed. affinity substance such as C60 [3, 4], organic dyes [5, 6] or nanoparticles [1, 7]. It has been successfully demonstrated that the photocurrent in these composite materials is typically increased by several orders of magnitude with regard to polymer-only devices $[1,2]$. The charge separation process must be fast compared to radiative decays of the singlet exciton, leading to quenching of the photoluminescence (PL) intensities. In this report, the microscopic mechanisms of exciton dissociation and migration in the MEH-PPV/CdSe nanoparticle hybrid materials were investigated by temperature dependent stead-state and time-resolved photoluminescence spectroscopy.

\section{Sample preparation and experimental set-up}

The CdSe nanoparticles were grown according to a procedure described in the literature [8]. In brief, $0.06 \mathrm{~g} \mathrm{CdO}, 5 \mathrm{~g}$ trioctylphosphine oxide (TOPO) and $0.26 \mathrm{~g}$ tetradecylphosphonic acid (TDPA) were loaded in a three-neck flask. At 

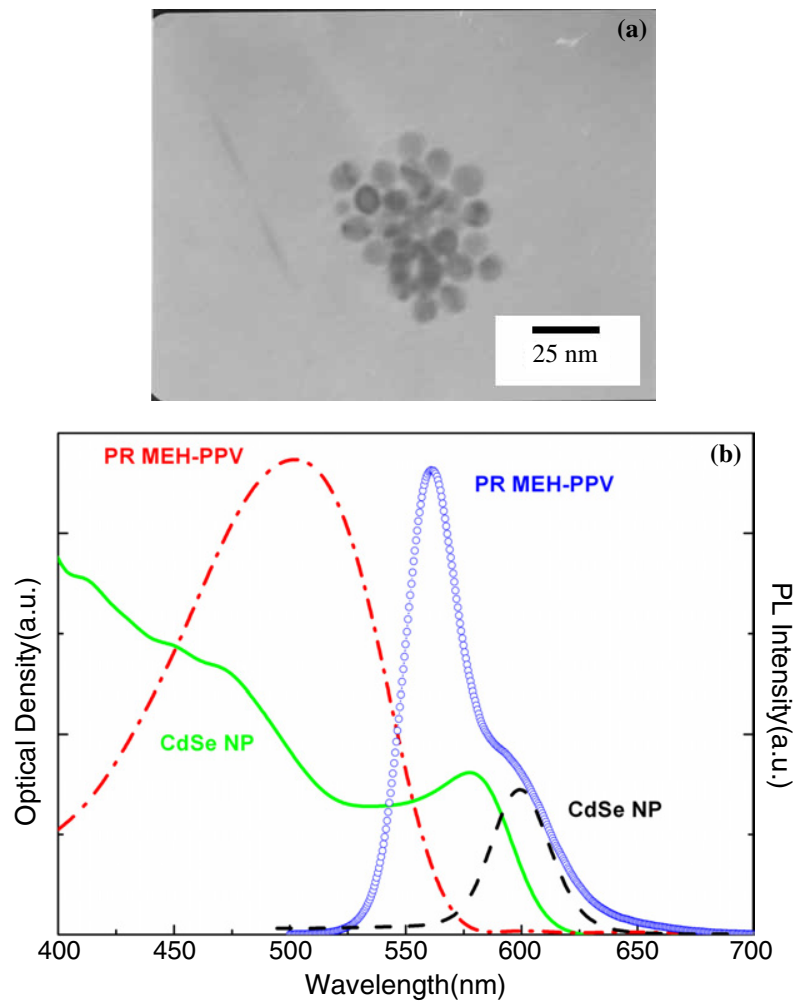

Figure 1. (a) TEM image of CdSe nanoparticles with a size of $6.0 \mathrm{~nm} \pm 10 \%$. (b) Absorption spectra of CdSe nanoparticles (solid line), pristine MEH-PPV (dash-dot line). The photoluminescence spectra of CdSe nanoparticles (dashed line) and pristine MEH-PPV (circle) are also shown.

about $300{ }^{\circ} \mathrm{C}$, the reddish $\mathrm{CdO}$ powder was dissolved into a colourless homogeneous solution. By cooling to $270^{\circ} \mathrm{C}$, followed by the injection of $0.9 \mathrm{ml}$ Se solution $(1.987 \mathrm{~g}$ Se dissolved in $50 \mathrm{ml}$ tributylphosphine (TBP)), high quality CdSe nanoparticles were obtained. The MEH-PPV polymer, with molecular weight $58000(\mathrm{Mn})$, was purchased from Aldrich Chemical company (USA). The polymer $(0.01 \mathrm{~g})$ was dissolved in toluene with a concentration of $1 \mathrm{wt} \%$. The hybrid materials were prepared by adding appropriate amounts of nanoparticles $(0.0025,0.067,0.015$ and $0.04 \mathrm{~g})$ into the $1 \mathrm{wt} \%$ polymer solution to make various ratios of nanoparticle/polymer composite samples.

UV-vis absorption spectra were obtained using an Ocean Optics HR-4000 spectrometer. The steady state PL spectra were obtained by pumping the samples with a continuous wave (CW) He-Cd laser (325 nm), and the emission spectra were analyzed using a Jobin-Yvon Triax $0.55 \mathrm{~m}$ monochromator and detected by a photomultiplier tube and standard photoncounting electronics. Time-resolved photoluminescence spectroscopy was performed with a time-correlated single photon counting (TCSPC) spectrometer (Picoquant, Inc.). A pulse laser $(375 \mathrm{~nm})$ with an average power of $1 \mathrm{~mW}$, operating at $40 \mathrm{MHz}$, with a duration of $70 \mathrm{ps}$ was used for excitation. During the temperature-dependent PL measurements, the samples were maintained under a vacuum inside a helium cryostat allowing temperature variation between $10 \mathrm{~K}$ and ambient temperature. The film thickness was measured using a Veeco M6 surface profiler.
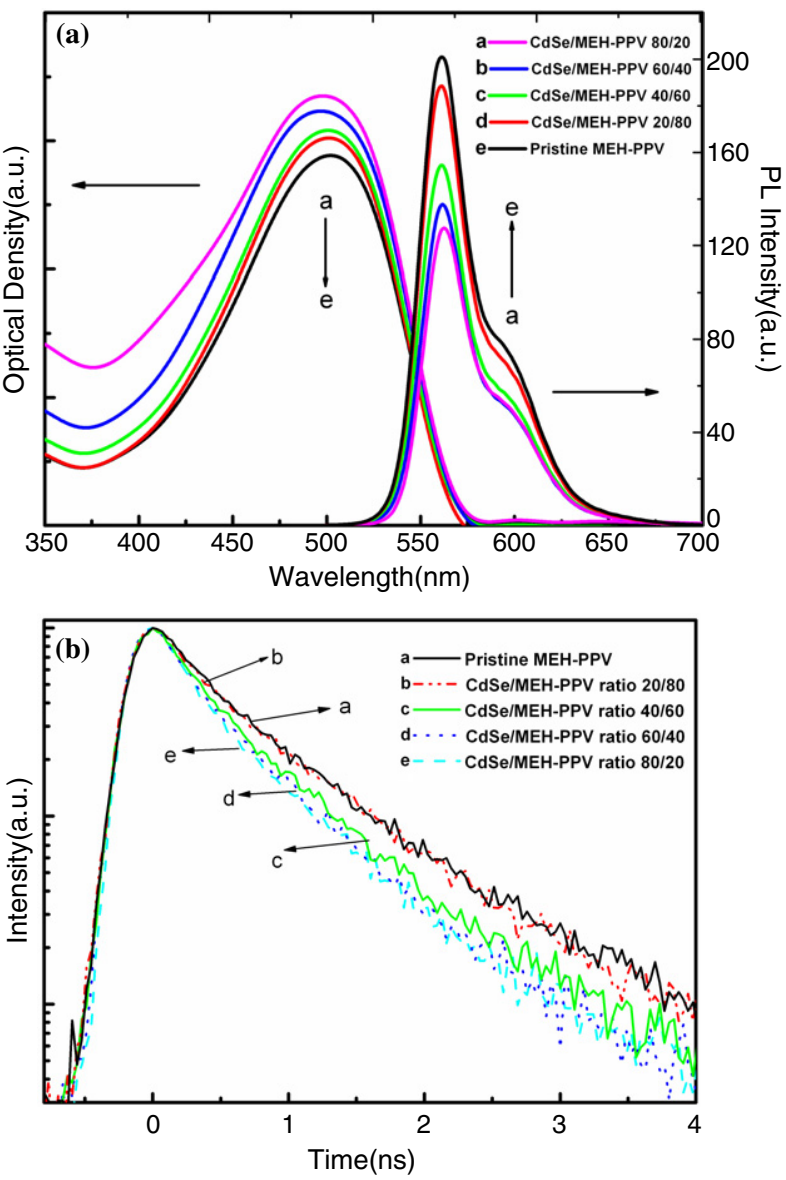

Figure 2. (a) Absorption and photoluminescence spectra of blends of MEH-PPV with CdSe nanoparticles for increasing concentrations of CdSe nanoparticles: (a) $0 \mathrm{wt} \%$, (b) $20 \mathrm{wt} \%$, (c) $40 \mathrm{wt} \%$, (d) $60 \mathrm{wt} \%$, and (e) $80 \mathrm{wt} \%$. (b) Photoluminescence lifetime decay of the above samples at room temperature.

\section{Results and discussions}

The inset of figure 1(a) shows the transmission electron microscopy (TEM) images of the CdSe nanoparticles before being mixed with MEH-PPV. The size of the nanoparticles was evaluated to be $6.0 \mathrm{~nm} \pm 10 \%$. The UV-vis spectrum of the CdSe nanoparticles shows a sharp absorption peak that indicates the nanoparticles are close to monodisperse. The excitonic absorption peak $(\sim 2.1 \mathrm{eV})$ as shown in figure $1(\mathrm{~b})$ exhibits a blue shift with respect to that of the bulk CdSe [9] due to the quantum confinement effect. For comparison, the representative absorption and PL spectra of the pristine MEHPPV in this study are also shown. All spectra in figure 1(b) have been normalized for clarity of presentation.

Figure 2(a) shows the room temperature absorption and PL spectra of the various blends of MEH-PPV with 0,20 , $40,60,80 \mathrm{wt} \%$ of CdSe nanoparticles, respectively. To avoid thickness and geometry effects, the optical density of absorption and PL intensity were measured in solution in cuvette cells. As the concentration of the CdSe nanoparticles increases from 20 to $80 \mathrm{wt} \%$, the optical density of the absorption spectra in the composites increases with respect to the pristine MEH-PPV, while the yield of the PL emission 
Table 1. Summary of the measured lifetime $\tau_{\mathrm{PL}}$ and the relative nature radiative lifetime for the pristine $\mathrm{MEH}-\mathrm{PPV}$ and the composites containing 20, 40, 60 and $80 \mathrm{wt} \%$ CdSe nanoparticles.

\begin{tabular}{lll}
\hline CdSe/MEH-PPV ratio & $\tau_{\mathrm{PL}}(\mathrm{ps})$ & $\tau_{\mathrm{R}}$ \\
\hline Pristine MEH-PPV & 450 & $\tau_{0}$ \\
20:80 (wt $\%)$ & 420 & $1.04 \tau_{0}$ \\
$40: 60$ & 350 & $1.11 \tau_{0}$ \\
$60: 40$ & 340 & $1.18 \tau_{0}$ \\
$80: 20$ & 330 & $1.21 \tau_{0}$ \\
\hline
\end{tabular}

decreases substantially, suggesting the occurrence of PL quenching in the composites. The PL spectra of the composites are found to be similar to that of the pristine MEH-PPV, indicating that the remaining luminescence predominantly results from excitons which radiatively recombine in the polymer due to the much lower PL efficiency of the CdSe nanoparticles compared to that of MEH-PPV [1]. The decrease in PL yield is attributed to quenching of the MEH-PPV PL emission by the CdSe nanoparticles acting as an electron accepting species, where significant charge separation takes place. Figure 2(b) presents the luminescence decays measured at $565 \mathrm{~nm}$. The PL decay in the composite films demonstrates a monotonic decrease in lifetime with increasing nanoparticle content. The measured lifetime of the luminescence $\tau_{\mathrm{PL}}$ can be related to the rate constants for radiative and nonradiative decays $k_{\mathrm{R}}$ and $k_{\mathrm{NR}}$ by

$$
\frac{1}{\tau_{\mathrm{PL}}}=k_{\mathrm{R}}+k_{\mathrm{NR}}
$$

The efficiency $\eta$ is then given by

$$
\eta=f \frac{k_{\mathrm{R}}}{k_{\mathrm{R}}+k_{\mathrm{NR}}}=f \frac{\tau_{\mathrm{PL}}}{\tau_{\mathrm{R}}},
$$

where $\tau_{\mathrm{R}}=1 / k_{\mathrm{R}}$ is the natural radiative lifetime i.e. the lifetime that the luminescence would have in the absence of competing non-radiative decay process. $f$ is the fraction of absorbed photons generating the emissive species. Analysis of the PL lifetime decay $\tau_{\mathrm{PL}}$ reveals a bi-exponential emission, summarized in table 1. As the nanoparticle concentration increases, a new relaxation process is present, which provides to the donor a further non-radiative process as the result of the separation of photogenerated electron-hole pairs. The enhancement of the non-radiative decay rate $k_{\mathrm{NR}}$ with increasing nanoparticle concentrations leads to shortening of the measured lifetime $\tau_{\mathrm{PL}}$ and quenching of the PL efficiency $\eta$. By taking the PL efficiency of the pristine MEH-PPV as 1 and the natural radiative lifetime as $\tau_{0}$, the relative natural radiative lifetime $\tau_{\mathrm{R}}$ for the composite films, which can be estimated from equations (1) and (2), are about 1.04, 1.11, 1.18 and $1.21 \tau_{0}$ for the samples containing 20, 40, 60 and $80 \mathrm{wt} \%$ nanoparticles, respectively. The increase in natural radiative lifetime $\tau_{R}$ for the composites with respect to the pristine MEH-PPV implies that the excitons, which contribute to the remaining luminescence by radiative recombination in the polymer, will migrate along the polymer chains over a larger region before recombination.

Figure 3 shows the photoresponse of the device comprising of the MEH-PPV/CdSe nanoparticle composite material between electrodes of ITO and aluminium. The

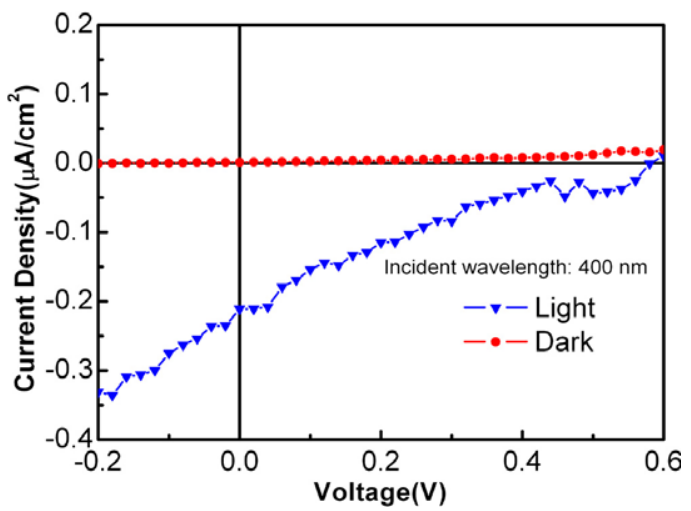

Figure 3. Current-voltage curve for a device containing $80 \mathrm{wt} \%$ $\mathrm{CdSe}$ in the dark (circle) and under illumination (triangle) at $400 \mathrm{~nm}$. The power density of the illumination is about $1.0 \mathrm{~W} \mathrm{~m}^{-2}$.
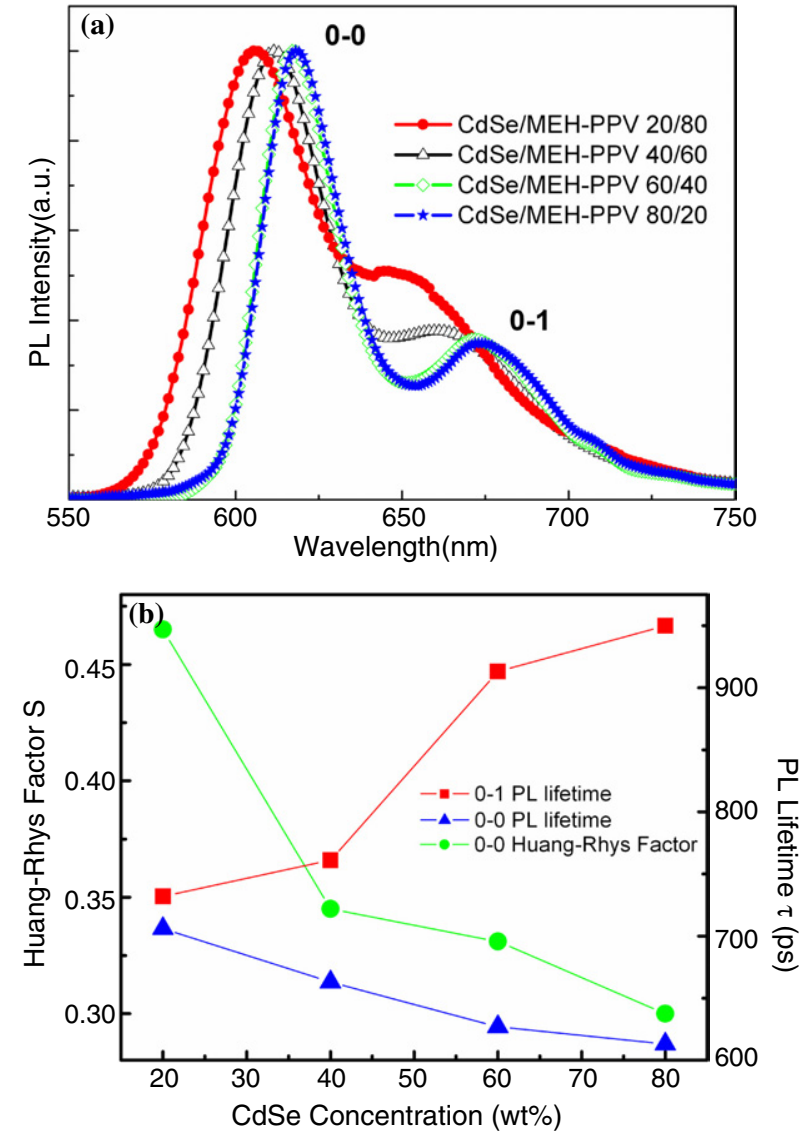

Figure 4. (a) Normalized photoluminescence spectra of $\mathrm{CdSe}$ nanoparticle/MEH-PPV hybrid materials measured at $10 \mathrm{~K}$. 0-0 and 0-1 peaks are ascribed to the zero-phonon line and the first vibronic band. (b) The Huang-Rhys factor $S, 0-0$ and $0-1$ photoluminescence decay time of the hybrid materials as a function of $\mathrm{CdSe}$ concentrations.

composite material consists of $80 \mathrm{wt} \%$ CdSe nanoparticles. The current-voltage curves of such a device, both in dark and under monochromatic illumination at $400 \mathrm{~nm}$, are shown. A significant increase in the photocurrent (at $0 \mathrm{~V}$ bias) for the device containing the MEH-PPV/CdSe nanoparticle composite material indicates that efficient charge separation takes place at the polymer/nanoparticle interfaces. 
Microscopic mechanisms for exciton migration in the polymer/nanoparticle composites can be further investigated by temperature dependent steady-state and time-resolved photoluminescence spectroscopy. Figure 4(a) shows the PL spectra of the composite films measured at $10 \mathrm{~K}$. The composite films were made by spin coating from the solutions containing various ratios of nanoparticle to polymer. The film thicknesses are 240, 256, 238, and $250 \mathrm{~nm}$ for the samples containing 20, 40, 60 and $80 \mathrm{wt} \%$ CdSe nanoparticles, respectively. The PL spectra in figure 4(a) consistently move toward a lower energy with respect to those in figure 2(a) measured at room temperature. The observed redshift in PL spectra results from an increase in the effective conjugated length while the temperature decreases due to the reduction in thermal disorder in the polymer. This enhances the delocalization of the $\pi$-electrons and lowers the band gap energy. The PL spectra were fitted with Gaussians in order to obtain the individual peak positions and line-width broadening parameters (full width of half maximum, FWHM). As the energy separation of the PL spectrum peaks corresponds to a phonon mode, the observed zero-phonon line (ZPL) and the first and second vibronic bands are labelled as 0$0,0-1$ and $0-2$, respectively. A progressive shift towards a lower energy, as well as the reduction in the FWHM in PL spectra can be observed by increasing CdSe nanoparticle content in the composite films. The dimensionless HuangRhys factor [10] $S$, described as $I_{0 \rightarrow n}=\frac{\mathrm{e}^{-S} S^{n}}{n !}$, where $n$ is the number of phonons involved, corresponding to the average number of phonons involved during relaxation, is directly related to the effective conjugated length of the polymer system. Figure 4(b) shows that $S$ monotonically decreases with increasing $\mathrm{CdSe}$ content as the result of enhanced conjugation in the polymer by reducing the disorder in conformation. The excited states are, therefore, delocalized over the more conjugated polymer chain, giving lower-energy emission. Longer conjugated sequences can be formed in the more rigid structures, owing to the inclusion of $\mathrm{CdSe}$ nanoparticles in the host polymer. Similar observations in poly(3-octylthiophene), consisting of thiophene rings with large alkyl groups, has also been reported [11]. The enhanced order in the composite films can be further evident from the time-resolved PL analysis. Figure 4(b) shows the PL lifetime decays of the $0-0$ and $0-1$ emission peaks measured at $10 \mathrm{~K}$ for the composite films with 20, 40, 60 and $80 \mathrm{wt} \%$ CdSe nanoparticles respectively. The PL lifetime decays for the dominant 0-0 emission decrease while those for the 0-1 emission increase with increasing CdSe nanoparticle content. The decreasing PL lifetime in the $0-0$ emission, as mentioned above, is attributed to efficient charge separation from the increase in the non-radiative pathway created by the inclusion of nanoparticles. The surviving excitons which contribute to the remaining luminescence in the polymer will migrate over more conjugated regions before recombination as a result of the increasing PL decay lifetime for the 0-1 emission peaks with increasing CdSe nanoparticle concentration. This result reflects the characteristics of enhanced conjugation in polymer/nanoparticle composite systems.

\section{Conclusion}

In conclusion, the mechanisms of exciton dissociation and migration in nanoparticle/polymer composites were investigated. Rapid charge separation occurs at the nanoparticle/polymer interfaces leading to efficient exciton dissociation, showing the quenching of the PL efficiency $\eta$ and the shortening of the PL lifetime $\tau_{\mathrm{PL}}$. The excitons which contribute to the remaining luminescence in the polymer migrate over more conjugated polymer chains, formed by the more rigid structures owing to the inclusion of $\mathrm{CdSe}$ nanoparticles in the host polymer.

\section{Acknowledgments}

This work is supported by the National Science Council, Taiwan (Project No. NSC93-2120-M-002-010) and the US Airforce project (Project No. AFOSR-AOARD-04-4023).

\section{References}

[1] Greenham N C, Peng X and Alivisatos A P 1996 Phys. Rev. B 5417628

[2] Huynh W U, Dittmer J J, Teclemariam N, Milliron D J and Alivisatos A P 2003 Phys. Rev. B 67115326

[3] Yu G and Heeger A J 2001 J. Appl. Phys. 784510

[4] Haugeneder A, Neges M, Kallinger C, Spirkl W, Lemmer U and Felmann J 1999 Phys. Rev. B 5915346

[5] Dittmer J et al 2000 Sol. Energy Mater. Sol. Cells 6153

[6] Dittmer J J, Marseglia E A and Friend R H 2000 Adv. Mater. 121270

[7] Warner J H, Watt A R, Thomsen E, Heckenberg N, Meredith P and Dunlop H R 2005 J. Phys. Chem. 1099001

[8] Chuang C-M, Lu W-B, Su W-F, Lin C-M and Chen Y-F 2005 J. Appl. Phys. 9796104

[9] Lifshitz E, Dag I, Litvin I, Holdes G, Gorer S, Reisfeld R, Zelner M and Minti H 1998 Chem. Phys. Lett. 288188

[10] Huang K and Rhys A 1950 Proc. R. Soc. 204406

[11] Watanabe A, Kodaira T and Ito O 1997 Chem. Phys. Lett. 273227 